\title{
Metformin therapy for the reproductive and metabolic consequences of polycystic ovary syndrome
}

\author{
Susan Sam ${ }^{1}$ - David A. Ehrmann ${ }^{1}$ \\ Received: 21 March 2017 / Accepted: 11 April 2017 /Published online: 3 August 2017 \\ (C) Springer-Verlag Berlin Heidelberg 2017
}

\begin{abstract}
Polycystic ovary syndrome (PCOS), the most common hormonal disorder among women of reproductive age, has various metabolic and reproductive consequences. Metformin was originally shown to lower testosterone levels in women with PCOS in the 1990s, an effect presumably related to its insulin sensitising actions. However, the precise mechanisms of metformin action in PCOS remain unclear and there is considerable heterogeneity in the clinical response to this therapy in women with PCOS. Recent evidence indicates that genetic factors may play a significant role in predicting response to metformin therapy in PCOS and future studies are needed to further identify women who are most likely to benefit from this therapy. At present, there is no clear evidence to support broad metformin use in PCOS. Well-designed prospective trials are needed to establish clear benefit for metformin use in the treatment of the reproductive and metabolic consequences associated with PCOS.
\end{abstract}

Keywords Body composition - Genetic mechanism • Hyperandrogenism $\cdot$ Insulin resistance $\cdot$ Lipid profile $\cdot$ Live birth $\cdot$ Menstrual irregularity $\cdot$ Polymorphisms $\cdot$ Review .

Testosterone $\cdot$ The metabolic syndrome

Electronic supplementary material The online version of this article (doi:10.1007/s00125-017-4306-3) contains a slide of the figure for download, which is available to authorised users.

Susan Sam

ssam@medicine.bsd.uchicago.edu

1 Department of Medicine, Section of Adult and Paediatric Endocrinology, Diabetes, and Metabolism, The University of Chicago, 5841 S. Maryland Avenue, MC1027, Chicago, IL 60637, USA

\begin{abstract}
Abbreviations
AMPK AMP-activated protein kinase

IMGD Insulin-mediated glucose disposal

PCOS Polycystic ovary syndrome
\end{abstract}

\section{Introduction}

Polycystic ovary syndrome (PCOS) was clinically characterised in 1935 based primarily upon its reproductive manifestations of menstrual irregularity and infertility, although hirsutism and obesity were also noted in this small cohort of women [1]. Over the ensuing decades, it became clear that this 'reproductive' syndrome was often accompanied by metabolic features that are a consequence of insulin resistance and hyperinsulinaemia [2-4], for example, impairment in oral glucose tolerance, type 2 diabetes $[5,6]$, the metabolic syndrome $[7,8]$ and dyslipidaemia $[9,10]$. The magnitude of insulin resistance in PCOS is greater than that caused by excess adiposity alone; it is characterised by reduced sensitivity and responsiveness to insulin-mediated glucose utilisation, primarily in the skeletal muscle, adipose tissue and in the liver [11]. Insulin resistance and resultant hyperinsulinaemia contribute to hyperandrogenaemia in PCOS by augmenting luteinising hormone-stimulated androgen production by ovarian theca cells [12-14] and by inhibiting hepatic synthesis of sex hormone-binding globulin (SHBG), the latter leading to an increase in bioavailable testosterone [15]. Weight loss or pharmacologic interventions that lower insulin levels have been shown to significantly reduce androgen levels in many women with PCOS [14].

In the 1990s, metformin was shown to ameliorate hyperandrogenism in both obese and non-obese women with PCOS. This effect was attributed to a reduction in hyperinsulinaemia because of enhanced insulin sensitivity 
$[16,17]$. Since these initial reports, there have been over 1000 original publications that have examined the effects of metformin in women with PCOS. Indeed, metformin is now among the most commonly prescribed medications in women with PCOS despite the fact that its use in this population is not approved by the Food and Drug Administration (FDA).

Although the mechanisms of metformin action are not well defined and the indications for its use are not clearly established, it appears that the salutary reproductive and metabolic effects of metformin in PCOS are both direct and indirect. This review provides an updated overview of metformin use in PCOS, encompassing our current understanding of its mechanisms of action.

\section{Mechanisms of action of metformin in PCOS}

\section{Role of genetic factors in metformin response}

There is significant variability in the clinical response to metformin treatment in PCOS. A meta-analysis of 38 RCTs of metformin use in 3495 women with PCOS revealed significant heterogeneity in its ability to reduce testosterone and insulin levels, regulate menses and improve body weight and composition $[18,19]$. This heterogeneity in response remained even after adjustment for many potential confounders. These findings suggest that unknown or unmeasured factor(s) impact the response to metformin therapy in PCOS. In type 2 diabetes, the glycaemic response to metfor$\mathrm{min}$ is heritable, although the genetic contribution is probably a result of individual variants across the genome rather than a few loci with large effect sizes [20]. Similarly, genetic factors are likely to mediate the response to metformin in PCOS.

Polymorphisms in genes involved in metformin transport or action have been implicated in the heterogeneous response to metformin in type 2 diabetes [21,22] and, to a lesser extent, in PCOS [23-27]. In a prospective randomised trial of over 300 women with PCOS, a polymorphism in the STK11 gene (expressed in the liver, also known as $L K B 1$ ), a serine threonine kinase that is required for metformin efficacy, was shown to be associated with a poor ovulatory response to metformin [26]. In type 2 diabetes, polymorphisms in the ATM gene, which regulates the activity of AMP-activated protein kinase (AMPK), a protein that plays a key role in cellular energy homeostasis [21], and in genes encoding metformin transport proteins, such as $O C T 1$, which is involved in the transport of metformin into hepatocytes [28], and $O C T 2$, which is involved in elimination of metformin through the renal tubule cells, are thought to play a role in metformin response. Similarly, in a study of 675 women with PCOS, $29.8 \%$ had non-functional alleles and $57.9 \%$ had low-functional alleles for polymorphisms in eight common SNPs on OCT1, OCT2 and ATM genes [24]. Two small European studies, demonstrating that polymorphisms in $O C T 1$ may account for variability of response to metformin in PCOS, support these findings [25, 27]. However, in a recent study, which included a larger sample size of women from North America, common variants in metformin transporter genes or ATM were not associated with decreases in glucose or testosterone levels, changes in body weight or improvements in glucose-mediated glucose disposal upon metformin use [23].

Thus, large-scale genome-wide studies are needed to identify markers that can predict metformin responsiveness in women affected with PCOS.

\section{Mechanisms of metformin action in target tissues relevant to PCOS}

Metformin acts on a number of tissues that have relevance to the metabolic and reproductive abnormalities in PCOS, namely the liver, skeletal muscle, adipose tissue and the ovary. Reduction in hepatic glucose output is the principal action of metformin although its mechanism(s) have not been clearly identified. The previously held view that metformin reduces glucose synthesis via activation of AMPK has been challenged; recent data indicate that lowered hepatic glucose output with metformin may result from inhibition of electron transport in mitochondrial respiratory Complex I [29] (this is described in more detail in the commentary by Rena et al in this issue of Diabetologia [30]), as well as antagonism of glucagon action in the liver [31].

Metformin suppresses lipolysis, modulates adipokine secretion and decreases lipogenesis in adipose tissue [32]. In skeletal muscle, metformin increases basal and insulinmediated glucose uptake possibly because of the reduction in glucotoxicity and lipotoxicity resulting from its actions in the liver and adipose tissue [32]. In the ovary, metformin is presumed to exert both direct and indirect effects on androgen production. Original studies demonstrated a reduction in CYP17 (also known as CYP17A1) activity in women with PCOS upon metformin treatment consequent to lowering of serum insulin levels [16]. However, there is evidence that metformin directly inhibits ovarian steroidogenesis [33, 34]. Inhibition of mitochondrial Complex I has been implicated as one potential mechanism for this action [35]. There is also evidence that metformin lowers androgen levels by an inhibitory effect on $3 \beta$-hydroxysteroid dehydrogenase $/ \Delta^{5}-\Delta^{4}$ isomerase type 2 [36].

\section{Impact of metformin on the clinical features of PCOS}

Over the past 20 years, metformin has been used to treat many of the metabolic and reproductive abnormalities associated with PCOS such as regulation of the menstrual cycle, induction of ovulation, and treatment of glucose intolerance and its related metabolic disorders. Yet, as aforementioned, whether 
metformin is of benefit in treating PCOS remains unclear [37, 38]. The majority of therapeutic studies have been small in size and have used metformin for a relatively brief period of time [33]. Hence, at present, a paucity of reliable clinical data does not permit firm indication for broad use of metformin to treat many of the reproductive and metabolic abnormalities associated with PCOS, as discussed below.

\section{Impact of metformin on reproductive features of PCOS}

Effects on androgens Metformin lowers testosterone levels by approximately 20 to $25 \%$ in women with PCOS [33]. This effect may be more pronounced among non-obese women with PCOS [18]. As discussed above, metformin is believed to lower testosterone levels by reducing hyperinsulinaemia [16, 17]. However, studies suggest other potential mechanisms for the testosterone-lowering actions of metformin in PCOS. Metformin reduces testosterone levels in PCOS within $48 \mathrm{~h}$ of therapy, prior to any significant changes in insulin sensitivity or other metabolic variables [34]. Brief exposure to metformin has also been reported to lead to lowering of testosterone levels in other studies [39]. Further, in one recent study, metformin lowered testosterone levels in women with PCOS despite the absence of any improvement in insulin sensitivity, as quantified by a frequently sampled IVGTT [40]. In vitro studies using cultured ovarian theca cells support a direct inhibitory effect of metformin on ovarian steroidogenesis [35, 41, 42] through inhibition of mitochondrial Complex I [35], a mechanism which has also been implicated in metformin action in the liver and other tissues [29, 31].

\section{Menstrual irregularity and clinical hyperandrogenism} Despite its testosterone-lowering actions, metformin use in PCOS is not consistently associated with improvements in menstrual irregularity or clinical hyperandrogenism. In a Cochrane review that included a meta-analysis of 38 randomised clinical trials of 3495 women with PCOS, metformin therapy only marginally improved menstrual pattern with significant heterogeneity in this measure [19]. In accordance with this finding, metformin improves ovulation rates in PCOS but with moderately high heterogeneity [19].

Additionally, metformin has not been shown to be an effective therapy for clinical symptoms of hyperandrogenism, such as acne or hirsutism [43]. Therefore, metformin is not recommended as a first-line treatment for management of menstrual irregularity or clinical hyperandrogenism in PCOS because of the lack of evidence for consistent benefits [37, 38, 44].

Fertility and live birth rate In a meta-analysis of randomised, placebo-controlled trials, metformin improved clinical pregnancy rates; however, it did not improve live birth rates, whether used alone or in combination with clomifene [19]. Similarly, in a more recent review consisting of 4227 women with PCOS, metformin only resulted in a marginal improvement in live birth rate when compared with placebo [45]. Therefore, metformin appears to have a limited role in improving fertility and live birth rate in women with PCOS and it is not recommended as a primary treatment for anovulatory infertility in this population $[37,38]$.

\section{Impact of metformin on metabolic features of PCOS}

Insulin sensitivity and hyperinsulinaemia The association between PCOS and hyperinsulinaemia was first reported by Burghen et al [2], who compared insulin responses during an OGTT in women with PCOS with a control group of women matched for BMI. Although the glucose response was similar between groups, insulin responses were significantly greater in women with PCOS [2]. Subsequent studies using the gold standard hyperinsulinaemic-euglycaemic clamp technique have clearly indicated a reduction in insulin-mediated glucose disposal (IMGD) in women with PCOS compared with ageand BMI matched women who were reproductively normal $[4,11]$. Treatment of women with PCOS with metformin for 6 to 12 months is associated with improvements in IMGD measured by hyperinsulinaemic-euglycaemic clamp technique [46, 47]. Based on these studies, metformin has been touted to be an 'insulin sensitiser'. As noted, metformin primarily acts to reduce hepatic glucose production [29, 31], which leads to a subsequent reduction in circulating insulin levels.

In contrast, studies in which frequently sampled IVGTTs have been used to quantify insulin sensitivity have not consistently shown significant improvements after treatment with metformin in women with PCOS [40, 48]. The reasons for the discordance in findings between methodologies are not well understood. However, in one of these studies, metformin improved glucose-mediated glucose disposal (i.e. glucose effectiveness) in PCOS [40]. This improved glucose effectiveness may account, at least in part, for the lower fasting glucose levels in response to metformin treatment in PCOS [19].

A large meta-analysis of 38 studies in women with PCOS revealed that metformin lowers fasting insulin levels but with significant heterogeneity. Sub-analysis based on BMI indicated a lowering of fasting insulin only among non-obese women with PCOS [19]. A meta-analysis of RCTs comparing the effect of metformin and lifestyle vs lifestyle alone did not demonstrate a significant improvement in fasting or $2 \mathrm{~h}$ insulin levels in women with PCOS with the addition of metformin to lifestyle interventions [49]. Furthermore, it is not clear if the addition of metformin to oral contraceptives has a significant impact on insulin levels in women with PCOS [50]. Hence, the clinical relevance of metformin use to improve insulin sensitivity in PCOS, and whether this treatment will lead to metabolic or cardiovascular benefits, is not clear at this time. 
Glucose tolerance Current guidelines for management of PCOS recommend metformin treatment for women with impaired glucose tolerance or type 2 diabetes who do not respond to lifestyle modification [37]. In type 2 diabetes, metformin is the recommended initial therapy and has also been demonstrated to reduce the risk of type 2 diabetes development in individuals with glucose intolerance [51]. However, studies investigating the impact of metformin on diabetes risk specifically in women with PCOS have not been performed. Nonetheless, considering that these women are at very high risk for developing diabetes $[5,6]$, it has been suggested that they will benefit from metformin therapy in case of glucose intolerance to reduce diabetes risk [33].

Body weight and composition The impact of metformin on weight loss and body composition has been examined in a number of observational and randomised clinical trials but the results have been conflicting. A meta-analysis of 16 randomised clinical trials of 630 participants treated with metformin for 6 months at an average daily dose of $1500 \mathrm{mg}$ reported no evidence of an effect on BMI and only a marginal reduction of waist:hip ratio [19]. However, there may be evidence for a beneficial effect of metformin on BMI and abdominal obesity when added to lifestyle modification. In a metaanalysis published in 2015, comparing nine randomised clinical trials ( $n=493$ participants) of metformin plus lifestyle vs lifestyle alone, metformin plus lifestyle was more effective in reducing BMI [49]. However, consistent with other metaanalyses [19], metformin alone was not associated with any reduction in BMI compared with lifestyle [49]. Very few studies have examined the impact of metformin on visceral vs subcutaneous fat depots. In one study, the addition of metformin to a low-calorie diet resulted in a modest impact on body weight and abdominal fat distribution in PCOS [52]. However, confirmation with larger well-designed randomised clinical trials is needed to clearly establish a benefit of metformin use in PCOS in this regard.

Lipid profile A meta-analysis of ten randomised clinical trials of 562 women with PCOS did not demonstrate an effect on serum cholesterol or triacylglycerol levels with metformin use [19]. However, it must be noted that the majority of the studies in this meta-analysis were small in sample size and brief in duration of metformin therapy, which may have impacted the final findings of the analysis.

Women with PCOS are at increased risk of cardiovascular disease as a result of the presence of insulin resistance and its related metabolic consequences, such as the metabolic syndrome, type 2 diabetes and dyslipidaemia. However, the actual prevalence of cardiovascular disease in PCOS is unknown. Whether metformin therapy can be used to reduce this risk is also currently not clear. Only well-designed, large-scale, prospective randomised clinical trials will be able to address these uncertainties.

\section{Conclusions}

Over the past few decades, metformin has been used to treat various abnormalities associated with PCOS despite the significant heterogeneity in clinical response and a lack of clear benefit (as summarised in Fig. 1). The conflicting findings related to the efficacy of metformin use in PCOS is partly related to the paucity of well-designed prospective trials of sufficient sample size with adequate duration of metformin use. Future studies are essential to identify predictors, such

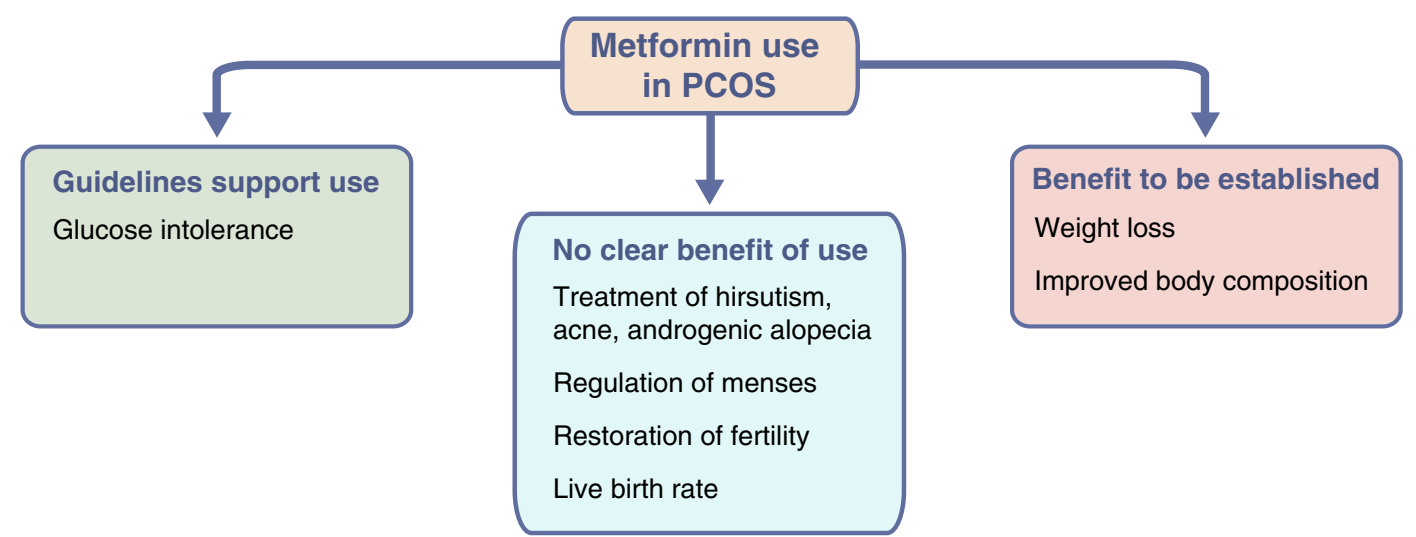

Fig. 1 Metformin use in PCOS. Metformin has been used to treat many of the reproductive and metabolic abnormalities associated with PCOS. However, evidence is lacking to support its broad use. Specifically, guidelines support the use of metformin for glucose intolerance in PCOS as it may prevent diabetes development in women with PCOS who are at high risk. In contrast, no clear benefit has been established to support metformin use for the reproductive features of PCOS, such as for the management of hyperandrogenic symptoms, regulation of menstrual cycles, or treatment of infertility and improvement in live birth rates 
as genetic factors, that forecast a beneficial response to metformin therapy in women with PCOS, especially with regards to many of its metabolic complications.

Funding Funding was provided by The Kovler Family Foundation.

Duality of interest The authors declare that there is no duality of interest associated with this manuscript.

Contribution statement Both authors were responsible for drafting the article and revising it critically for important intellectual content. All authors approved the version to be published.

\section{References}

1. Stein I, Leventhal M (1935) Amenorrhea associated with bilateral polycystic ovaries. Am J Obstet Gynecol 29:181-191

2. Burghen GA, Givens JR, Kitabchi AE (1980) Correlation of hyperandrogenism with hyperinsulinism in polycystic ovarian disease. J Clin Endocrinol Metab 50:113-116

3. Chang RJ, Nakamura RM, Judd HL, Kaplan SA (1983) Insulin resistance in nonobese patients with polycystic ovarian disease. J Clin Endocrinol Metab 57:356-359

4. Dunaif A, Segal KR, Futterweit W, Dobrjansky A (1989) Profound peripheral insulin resistance, independent of obesity, in polycystic ovary syndrome. Diabetes 38:1165-1174

5. Legro RS, Kunselman AR, Dodson WC, Dunaif A (1999) Prevalence and predictors of risk for type 2 diabetes mellitus and impaired glucose tolerance in polycystic ovary syndrome: a prospective, controlled study in 254 affected women. J Clin Endocrinol Metab 84:165-169

6. Ehrmann DA, Barnes RB, Rosenfield RL, Cavaghan MK, Imperial J (1999) Prevalence of impaired glucose tolerance and diabetes in women with polycystic ovary syndrome. Diabetes Care 22:141146

7. Apridonidze T, Essah PA, Iuorno MJ, Nestler JE (2005) Prevalence and characteristics of the metabolic syndrome in women with polycystic ovary syndrome. J Clin Endocrinol Metab 90:1929-1935

8. Ehrmann DA, Liljenquist DR, Kasza K, Azziz R, Legro RS, Ghazzi MN (2006) Prevalence and predictors of the metabolic syndrome in women with polycystic ovary syndrome. J Clin Endocrinol Metab 91:48-53

9. Legro RS, Kunselman AR, Dunaif A (2001) Prevalence and predictors of dyslipidemia in women with polycystic ovary syndrome. Am J Med 111:607-613

10. Talbott E, Clerici A, Berga SL et al (1998) Adverse lipid and coronary heart disease risk profiles in young women with polycystic ovary syndrome: results of a case-control study. J Clin Epidemiol 51:415-422

11. Dunaif A, Segal KR, Shelley DR, Green G, Dobrjansky A, Licholai T (1992) Evidence for distinctive and intrinsic defects in insulin action in polycystic ovary syndrome. Diabetes 41:1257-1266

12. Dunaif A, Graf M (1989) Insulin administration alters gonadal steroid metabolism independent of changes in gonadotropin secretion in insulin-resistant women with the polycystic ovary syndrome. J Clin Invest 83:23-29

13. Micic D, Popovic V, Nesovic M et al (1988) Androgen levels during sequential insulin euglycemic clamp studies in patients with polycystic ovary disease. J Steroid Biochem 31:995-999
14. Nestler JE, Barlascini CO, Matt DW et al (1989) Suppression of serum insulin by diazoxide reduces serum testosterone levels in obese women with polycystic ovary syndrome. J Clin Endocrinol Metab 68:1027-1032

15. Nestler JE, Powers LP, Matt DW et al (1991) A direct effect of hyperinsulinemia on serum sex hormone-binding globulin levels in obese women with the polycystic ovary syndrome. J Clin Endocrinol Metab 72:83-89

16. Nestler JE, Jakubowicz DJ (1996) Decreases in ovarian cytochrome P450c17 alpha activity and serum free testosterone after reduction of insulin secretion in polycystic ovary syndrome. N Engl J Med 335:617-623

17. Nestler JE, Jakubowicz DJ (1997) Lean women with polycystic ovary syndrome respond to insulin reduction with decreases in ovarian P450c17 alpha activity and serum androgens. J Clin Endocrinol Metab 82:4075-4079

18. Tang T, Lord JM, Norman RJ, Yasmin E, Balen AH (2010) Insulinsensitising drugs (metformin, rosiglitazone, pioglitazone, D-chiroinositol) for women with polycystic ovary syndrome, oligo amenorrhoea and subfertility. Cochrane Database Syst Rev, Issue 1, Art. no.:CD003053. DOI: 10.1002/14651858.CD003053.pub4

19. Tang T, Lord JM, Norman RJ, Yasmin E, Balen AH (2012) Insulinsensitising drugs (metformin, rosiglitazone, pioglitazone, D-chiroinositol) for women with polycystic ovary syndrome, oligo amenorrhoea and subfertility. Cochrane Database Syst Rev, Issue 5, Art. no.:CD003053. DOI: 10.1002/14651858.CD003053.pub5

20. Zhou K, Donnelly L, Yang J et al (2014) Heritability of variation in glycaemic response to metformin: a genome-wide complex trait analysis. Lancet Diabetes Endocrinol 2:481-487

21. Zhou K, Yee SW, Seiser EL et al (2016) Variation in the glucose transporter gene SLC2A2 is associated with glycemic response to metformin. Nat Genet 48:1055-1059

22. Jablonski KA, McAteer JB, de Bakker PI et al (2010) Common variants in 40 genes assessed for diabetes incidence and response to metformin and lifestyle intervention in the diabetes prevention program. Diabetes 59:2672-2681

23. Pau CT, Cheang KI, Modi BP et al (2016) The role of variants regulating metformin transport and action in women with polycystic ovary syndrome. Pharmacogenomics 17:1765-7173

24. Schweighofer N, Lerchbaum E, Trummer O, Schwetz V, Pieber T, Obermayer-Pietsch B (2014) Metformin resistance alleles in polycystic ovary syndrome: pattern and association with glucose metabolism. Pharmacogenomics 15:305-317

25. Gambineri A, Tomassoni F, Gasparini DI et al (2010) Organic cation transporter 1 polymorphisms predict the metabolic response to metformin in women with the polycystic ovary syndrome. J Clin Endocrinol Metab 95:E204-E208

26. Legro RS, Barnhart HX, Schlaff WD et al (2008) Ovulatory response to treatment of polycystic ovary syndrome is associated with a polymorphism in the STK11 gene. J Clin Endocrinol Metab 93:792-800

27. Diaz M, Lopez-Bermejo A, Sanchez-Infantes D, Bassols J, de Zegher F, Ibanez L (2011) Responsiveness to metformin in girls with androgen excess: collective influence of genetic polymorphisms. Fertil Steril 96:208-213

28. Shu Y, Brown C, Castro RA et al (2008) Effect of genetic variation in the organic cation transporter 1, OCT1, on metformin pharmacokinetics. Clin Pharmacol Ther 83:273-280

29. El-Mir MY, Nogueira V, Fontaine E, Averet N, Rigoulet M, Leverve X (2000) Dimethylbiguanide inhibits cell respiration via an indirect effect targeted on the respiratory chain complex I. J Biol Chem 275:223-228

30. Rena G, Hardie DG, Pearson ER (2017) The mechanisms of action of metformin. Diabetologia. DOI:10.1007/s00125-017-4342-Z

31. Miller RA, Chu Q, Xie J, Foretz M, Viollet B, Birnbaum MJ (2013) Biguanides suppress hepatic glucagon signalling by decreasing production of cyclic AMP. Nature 494:256-260 
32. Diamanti-Kandarakis E, Christakou CD, Kandaraki E, Economou FN (2010) Metformin: an old medication of new fashion: evolving new molecular mechanisms and clinical implications in polycystic ovary syndrome. Eur J Endocrinol 162:193-212

33. McCartney CR, Marshall JC (2016) Polycystic ovary syndrome. N Engl J Med 375:54-64

34. Kurzthaler D, Hadziomerovic-Pekic D, Wildt L, Seeber BE (2014) Metformin induces a prompt decrease in LH-stimulated testosterone response in women with PCOS independent of its insulinsensitizing effects. Reprod Biol Endocrinol 12:1-6

35. Hirsch A, Hahn D, Kempna P et al (2012) Metformin inhibits human androgen production by regulating steroidogenic enzymes HSD3B2 and CYP17A1 and complex I activity of the respiratory chain. Endocrinology 153:4354-4366

36. Lee BH, Indran IR, Tan HM et al (2016) A dietary medium-chain fatty acid, decanoic acid, inhibits recruitment of Nur77 to the HSD3B2 promoter in vitro and reverses endocrine and metabolic abnormalities in a rat model of polycystic ovary syndrome. Endocrinology 157:382-394

37. Legro RS, Arslanian SA, Ehrmann DA et al (2013) Diagnosis and treatment of polycystic ovary syndrome: an Endocrine Society clinical practice guideline. J Clin Endocrinol Metab 98:4565-4592

38. Goodman NF, Cobin RH, Futterweit W et al (2015) American Association of Clinical Endocrinologists, American College of Endocrinology, and Androgen Excess and PCOS Society disease state clinical review: guide to the best practices in the evaluation and treatment of polycystic ovary syndrome - part 1 . Endocr Pract 21:1291-1300

39. Pirwany IR, Yates RW, Cameron IT, Fleming R (1999) Effects of the insulin sensitizing drug metformin on ovarian function, follicular growth and ovulation rate in obese women with oligomenorrhoea. Hum Reprod 14:2963-2968

40. Pau CT, Keefe C, Duran J, Welt CK (2014) Metformin improves glucose effectiveness, not insulin sensitivity: predicting treatment response in women with polycystic ovary syndrome in an openlabel, interventional study. J Clin Endocrinol Metab 99:1870-1878

41. Mansfield R, Galea R, Brincat M, Hole D, Mason H (2003) Metformin has direct effects on human ovarian steroidogenesis. Fertil Steril 79:956-962
42. Attia GR, Rainey WE, Carr BR (2001) Metformin directly inhibits androgen production in human thecal cells. Fertil Steril 76:517-524

43. Cosma M, Swiglo BA, Flynn DN et al (2008) Clinical review: insulin sensitizers for the treatment of hirsutism: a systematic review and metaanalyses of randomized controlled trials. J Clin Endocrinol Metab 93:1135-1142

44. Martin KA, Chang RJ, Ehrmann DA et al (2008) Evaluation and treatment of hirsutism in premenopausal women: an endocrine society clinical practice guideline. J Clin Endocrinol Metab 93:1105-1120

45. Balen AH, Morley LC, Misso M et al (2016) The management of anovulatory infertility in women with polycystic ovary syndrome: an analysis of the evidence to support the development of global WHO guidance. Hum Reprod Update 22:687-708

46. Diamanti-Kandarakis E, Kouli C, Tsianateli T, Bergiele A (1998) Therapeutic effects of metformin on insulin resistance and hyperandrogenism in polycystic ovary syndrome. Eur J Endocrinol 138:269-274

47. Palomba S, Falbo A, Russo T et al (2007) Insulin sensitivity after metformin suspension in normal-weight women with polycystic ovary syndrome. J Clin Endocrinol Metab 92:3128-3135

48. Ehrmann DA, Cavaghan MK, Imperial J, Sturis J, Rosenfield RL, Polonsky KS (1997) Effects of metformin on insulin secretion, insulin action, and ovarian steroidogenesis in women with polycystic ovary syndrome. J Clin Endocrinol Metab 82:524-530

49. Naderpoor N, Shorakae S, de Courten B, Misso ML, Moran LJ, Teede HJ (2015) Metformin and lifestyle modification in polycystic ovary syndrome: systematic review and meta-analysis. Hum Reprod Update 21:560-574

50. Costello MF, Shrestha B, Eden J, Johnson NP, Sjoblom P (2007) Metformin versus oral contraceptive pill in polycystic ovary syndrome: a Cochrane review. Hum Reprod 22:1200-1299

51. Knowler WC, Barrett-Connor E, Fowler SE et al (2002) Reduction in the incidence of type 2 diabetes with lifestyle intervention or metformin. N Engl J Med 346:393-403

52. Pasquali R, Gambineri A, Biscotti D et al (2000) Effect of long-term treatment with metformin added to hypocaloric diet on body composition, fat distribution, and androgen and insulin levels in abdominally obese women with and without the polycystic ovary syndrome. J Clin Endocrinol Metab 85:2767-2774 